https://helda.helsinki.fi

\title{
Skin biomes
}

\section{Fyhrquist, Nanna}

2016-05

Fyhrquist , N , Salava , A , Auvinen , P \& Lauerma , A 2016 , ' Skin biomes ' , Current Allergy and Asthma Reports, vol. 16 , no. 5 , 27056560 , pp. 40 .

https://doi.org/10.1007/s11882-016-0618-5, https://doi.org/10.1007/s11882-016-0618-5

http://hdl.handle.net/10138/223991

https://doi.org/10.1007/s11882-016-0618-5

publishedVersion

Downloaded from Helda, University of Helsinki institutional repository.

This is an electronic reprint of the original article.

This reprint may differ from the original in pagination and typographic detail.

Please cite the original version. 


\title{
Skin Biomes
}

\author{
N. Fyhrquist $^{1}$ - A. Salava ${ }^{2} \cdot$ P. Auvinen ${ }^{3} \cdot$ A. Lauerma $^{2}$
}

Published online: 7 April 2016

(C) Springer Science+Business Media New York 2016

\begin{abstract}
The cutaneous microbiome has been investigated broadly in recent years and some traditional perspectives are beginning to change. A diverse microbiome exists on human skin and has a potential to influence pathogenic microbes and modulate the course of skin disorders, e.g. atopic dermatitis. In addition to the known dysfunctions in barrier function of the skin and immunologic disturbances, evidence is rising that frequent skin disorders, e.g. atopic dermatitis, might be connected to a dysbiosis of the microbial community and changes in the skin microbiome. As a future perspective, examining the skin microbiome could be seen as a potential new diagnostic and therapeutic target in inflammatory skin disorders.
\end{abstract}

Keywords Skin microbiome $\cdot$ Metagenomics $\cdot$ Cutaneous microbial diversity $\cdot$ Inflammatory skin diseases .

Staphylococcus aureus

This article is part of the Topical Collection on Allergic Skin Diseases

A. Lauerma

antti.lauerma@hus.fi

1 Department of Bacteriology and Immunology, University of Helsinki, Helsinki, Finland

2 Department of Dermatology and Allergology, University of Helsinki and Helsinki University, Meilahdentie 2, 00250 Helsinki, Finland

3 Institute of Biotechnology, DNA Sequencing and Genomics Laboratory, University of Helsinki, Helsinki, Finland

\section{Introduction}

The human body is home to trillions of microorganisms, which outnumber human cells by a factor of ten and human genes by a factor of 100 . Multicellular organisms and microbes likely have co-evolved since ancient times, with the host providing a living habitat, nutrients and shelter, and microorganisms contributing essentially to host metabolism and immunity [1•]. The development of the immune system, and in particular adaptive immunity, has coincided with the acquisition of a complex microbiota, suggesting that defined arms of the immune system evolved to maintain a symbiotic relationship. However, this alliance comes at a price where external factors such as diet or the use of antibiotics may change rapidly, causing significant changes in the microbiota, and as a direct result changes in the immune system, which are now believed to contribute to the rapid increase in chronic inflammatory disorders seen in high-income countries [2••].

The bulk of the microbiota associated with the human body resides in the gut, while only a minor share inhabit epithelial surfaces such as the mouth, upper and lower airways, vagina and skin. The skin is a $2-\mathrm{m}^{2}$ large epithelial surface inhabited by $10^{6}$ bacteria per square centimeter, mounting up to a total of $10^{12}$ skin microbes. The relative scarcity of microbes on the skin is a consequence of the highly inhospitable environment, including variable temperatures, a generally low $\mathrm{pH}$ and lack of nutrients, high levels of salt and antimicrobial molecules, and arbitrary exposure to sun, wind and other external factors. Nevertheless, there is a core population of microbes that thrive on the skin, with a highly variable element of transient inhabitants $[3 \cdot \bullet]$.

Before exceedingly efficient molecular techniques became available, the human skin microbiome was investigated by culturing methods, which introduced a bias with regard to species that grow versus species that do not grow in laboratory 
conditions. The new techniques, including $16 \mathrm{~S}$ ribosomal RNA (rRNA) sequencing and metagenomics shotgun sequencing, allow for direct identification of microbes in the sample but do not discriminate between dead and alive organisms. 16S rRNA sequencing is restricted to taxonomic identification of bacteria, while the metagenomics approach, which explores the entire genomes of the microbiota, extends the analysis from bacteria to include viruses, fungi and archae. Furthermore, metagenomics shotgun sequencing allows for the identification of microbes at the strain level, as well as the analysis of functional potential $[3 \bullet \bullet, 4 \bullet]$. Ideally, combining shotgun metagenomics with metatranscriptomics, i.e. analysis of microbial gene expression, as well as metabolomics, allows for identification of the actual functional activity of the microbiota [5]. However, these analyses are seriously challenged by the scarcity of microbial DNA generally available in skin samples, and the usually high level of human DNA contamination. Moreover, the enormous amount of data collected by these methods is a challenge in itself, while the processing and analysis of data is far from standardized, and available databases and analytical tools are limited.

The skin provides a boundary between the body and the environment, restricting water loss and preventing entry of harmful substances and microorganisms, and providing a first line of defense against infection. Immune responses in the skin are regulated by an extensive crosstalk between epithelial, stromal and immune cells to ensure effective host defense and tissue homeostasis. The microbes that colonize the skin are in constant interaction with the host cells, thereby influencing host immunity, and the maintenance of immune balance depends on a finely tuned equilibrium of interactions between the host and microbiota [6]. Recently, it was discovered that bacteria are not just present on the surface of the skin but can penetrate the outer skin barrier and interact with cells deeper in the dermis, and thereby the capacity of the skin microbiome to influence immune homeostasis is even more apparent [7].

\section{The Skin Ecosystem}

The conditions on the skin vary with skin thickness, folds and the density of hair follicles and glands. Sweat glands (eccrine and apocrine glands), sebaceous glands and hair follicles provide their unique microenvironments. Eccrine glands continuously bath the skin surface with sweat containing mostly water and salt, while sebaceous glands secrete sebum which protects and lubricates the skin and provides an antibacterial shield. Depending on the topography of the skin, there are areas that are partially occluded and higher in temperature and humidity (groin, axillary vault and toe web), as opposed to highly desiccated areas (leg and arm) which are subjected to large surface temperature fluctuations. Moreover, there are areas with a high density of sebaceous glands (face, chest and back) providing a sebum-rich environment. Skin physiology mainly determines the pattern of colonization by skin microbes, with Staphylococcus spp., Corynebacterium spp. being the most abundant microorganisms in moist sites, and lipophilic microorganisms such as Propionibacterium spp. and Malassezia spp. dominating sebaceous areas. Dry areas are the most diverse sites with a mixed representation of organisms including Actinobacteria, Proteobacteria, Firmicutes and Bacteroidetes, and an unexpectedly high abundance of gram-negative bacteria [3・•] (Fig. 1).

The skin is colonized by a handful of stable inhabitants, with rare or transient species contributing to interpersonal variation. Most skin bacteria fall into four phyla: Actinobacteria, Firmicutes, Bacteroidetes and Proteobacteria. The same phyla are found also on inner mucosal surfaces, but unlike in the gut, which is dominated by Firmicutes and Bacteroidetes, the skin is colonized most abundantly by Actinobacteria. Compared with the gut environment, the skin is remarkably poor in nutrients, providing only sweat, sebum and constituents of the stratum corneum as sources of nutrients. The central components of sweat are urea, proteins, lactic acid and a wide range of amino acids [8] while human sebum consists of triglycerides and fatty acids, wax esters and squalene [9]. The stratum corneum contains mainly protein and lipids, besides water and a small quantity of other substances. Other challenges are the acidic $\mathrm{pH}(\sim 5)$ of the skin surface, the high salt content, and abundant antibacterial molecules such as free fatty acids, sphingosine, nitric oxide, immunoglobulins and antimicrobial peptides.

Nevertheless, there are microbes which thrive on the skin. One of the most prevalent microbes on the skin is gram-positive Staphylococcus epidermidis, which is a member of the Firmicutes. Staphylococci are highly adaptable with a preference for occluded and moist skin sites such as the axilla and the groin, but they are also found on drier and more exposed sites such as the volar forearm. Staphylococci can adapt well to conditions of high salinity and may utilize urea in sweat as a source of nitrogen. Furthermore, Staphylococci express adhesins which may facilitate adherence to the skin and proteases which may contribute to the remodeling of the stratum corneum and liberation of nutrients.

Gram-positive Corynebacteria prefer to grow on moist or sebaceous skin sites and are frequently lipohilic (lipid auxotrophs) and therefore do not produce their own lipids, but utilize the lipid contents of sebum and the 
Fig. 1 Mean relative abundances of the seven most common genera of the cutaneous microbiome in different microenvironments of the human skin. From Sequence

Read Archive at the European

Bioinformatics Institute (accession no. PRJEB7554) [30]

Sebaceus microenvironment (chest, neckline)

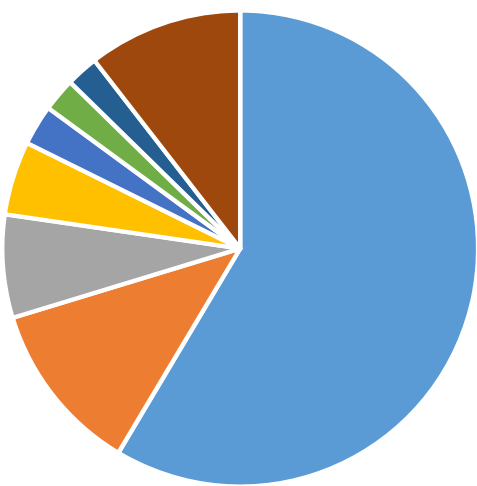

\footnotetext{
- Proprionibacteria spp. " Coruynebacterium spp = Lactococcus spp. $\quad$ Staphylococcus spp.

- Prevottella spp. - Enhydrobacter spp. - - Streptococcus spp. - Other taxa
}

\section{Moist microenvironment (armpit)}

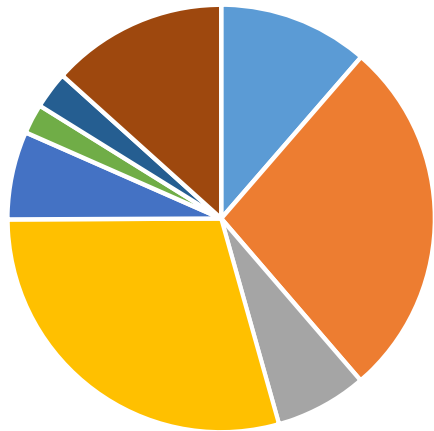

- Proprionibacterium spp. - Corynebacterium spp = Lactococcus spp.

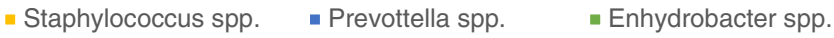

- Streptococcus spp. = Other taxa

\section{Dry microenvironment (lower back)}

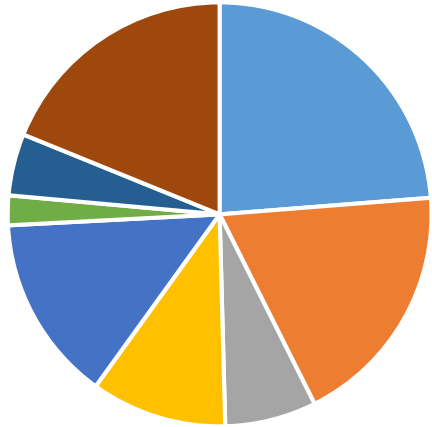

\footnotetext{
- Proprionibacterium spp. $=$ Corynebacterium spp = Lactococcus spp.

- Staphylococcus spp. - - Prevottella spp. = Enhydrobacter spp.

- Streptococcus spp. = Other taxa
}

stratum corneum. Moreover, most Corynebacteria are capable of growing in the presence of high salt concentrations and therefore able to colonize sites rich in eccrine glands.
The slow-growing Propionibacteria are gram-positive, anaerobic bacilli, with a general preference for skin areas rich in sebum, and some species preferring areas with a high density of eccrine glands. Propionibacteria 
tend to localize to hair follicles, which provide an environment with low oxygen tension. Propionibacteria acnes is the best known member of this genus and capable of acquiring nutrients from sebum thanks to a multitude of lipases. P. acnes also produces proteases capable of liberating amino acids from skin proteins.

\section{Microbe-Microbe Dynamics}

The community composition of the skin microbiota is continuously influenced by the availability of nutrients and space, the level of antimicrobial molecules produced by the host and other microbes, as well as by host immune responses. The human-associated microbiota produces several small molecules, including ribosomally synthesized, posttranslationally modified peptides (RiPPs), to mediate these competitive and social interactions. These molecules generally have a narrow spectrum activity and mediate interactions among closely related species. They are toxic for a limited set of species closely related to the producer and likely determine niche colonization. RiPPs include numerous subclasses, including lantibiotics, bacteriocins, microcins, thiaxole/oxazole-modified microcins and thiopeptides. Lantibiotics are short peptides of less than 40 amino acids that contain thioether bonds, which are redox-stable and the modified amino acids form ring structures that are important for bacteriocidal activities such as pore formation in bacterial cell membranes. Predominately, the Firmicutes produce lantibiotics, for instance $S$. epidermidis produces the structurally unique lantibiotics epidermin, pep5 and epilancin K7 and Staphylococcus aureus produces Au-26 (also known as Bsa) which inhibits the growth of Lactobacilli [10]. Thus, both commensals and pathogens use lantibiotics to compete and establish colonization.

In contrast to lantibiotics, bacteriocins are longer peptides and usually unmodified. S. epidermidis produces phenol soluble modulins (PSM), which are amphiphatic molecules with clusters of positively charged and hydrophobic amino acid chains, allowing for interaction with negatively charged phospholipid head groups and hydrophobic fatty acid chains in microbial membranes, resulting in pore formation. S. epidermidis derived PSMs selectively kill pathogens such as $S$. aureus, group A Streptococcus and Escherichia coli, providing an important means of resisting colonization by opportunistic or pathogenic microorganisms.

Moreover, S. epidermidis is capable of inhibiting nasal colonization by $S$. aureus through the production of serine protease which destroys biofilms and inhibits biofilm formation and $S$. epidermidis can block the $S$. aureus quorum sensing system by producing a thiolactone-containing peptide [11].
Thus, commensals like $S$. epidermidis may contribute actively to the skin immune defense by supplying additional antimicrobial molecules (AMPs) which act together with hostderived AMPs.

\section{Microbe-Host Interplay}

The microbial communities on the skin might be key players in host defense. Human commensal skin bacteria may both directly protect humans from pathogenic invaders and help the immune system to maintain a balance between protection and inflammation. In addition to being a physical barrier, the skin contains an active immune barrier responding to wounding and infection and also modulating the commensal microbiota that colonize the skin. Keratinocytes and cells of the innate immune system continuously sample the microbiota colonizing the skin through pattern recognition receptors such as toll-like receptors, mannose receptors and NOD-like receptors. These receptors recognize various components of the microorganisms and initiate innate immune responses resulting in the secretion of AMPs, cytokines and chemokines, which in turn activate and tune adaptive immune responses. How skin commensal products are recognized by the immune system are largely unknown, but one could speculate that dendritic cells surrounding the microbial communities may be able to directly capture microbes or microbial products, and microbial metabolites may be able to diffuse through the skin and be captured and sensed by neighboring cells.

Recent studies have demonstrated that symbiotic factors produced by commensal bacteria beneficially modulate the host immune system, decreasing the risk of autoimmune and allergic inflammatory diseases. Capsular polysaccharides from Bacteroides and Streptococcus are not simply structural or nonspecifically adhesive, but they may also have highly specific ligand-receptor interactions that result in immune modulation. Polysaccharide A of Bacteroides fragilis is known to signal to the host's innate immune system through toll-like receptor 2 (TLR2), which then leads to the induction of $\mathrm{T}$ regulatory cells, which produce IL-10 and restrict the activity of TH17 cells and suppress H. hepaticus induced colitis [12]. Likewise, short chain fatty acids (SCFAs), which are products of dietary fiber fermentation by intestinal microbiota, reduce the activity and recruitment of neutrophils through GPR43-dependent mechanisms [13] and contribute to the suppression of colitis, arthritis and airway inflammation in mouse models.

In the skin, these aspects have been much less investigated, but there are elements of the resident microbiota which influence cutaneous immune systems and hostpathogen interactions. The SCFAs acetate, propionate 
and butyrate which are main metabolic products of fermentation by anaerobic bacteria in the intestine, are important regulators of intestinal immune functions. SCFAs act on leukocytes and endothelial cells through the activation of GPCRs and inhibition of histone deacetylate. SCFAs regulate leukocyte activities including the production of cytokines and chemokines and migration to sites of inflammation to destroy microbial pathogens. P. acnes which is widely distributed over the skin surface, resides also deeper in the skin in hair follicles and glands, where the anaerobic environment may trigger $P$. acnes to undergo fermentation turning carbon sources such as glycerol or glucose into propionic acid, which has known antimicrobial activity [14] and significantly suppresses the growth of $S$. aureus [15]. Both the fermentation process and a capacity of $P$. acnes to release fatty acids onto the skin by hydrolyzing sebum triglycerides lowers the $\mathrm{pH}$ of the skin environment, which inhibits the growth of common pathogens such as S. aureus and S. pyogenes. Moreover, free fatty acids (such as lauric acid, palmitic acid and oleic acid) have direct antibacterial activity against a broad range of gram-positive bacteria and enhance the skin innate immune defense by inducing the production of hBDs in sebocytes [16]. Moreover, S. epidermidisderived small molecules trigger the production of hBDs and RNASe7 in keratinocytes via TLR2mediated mechanisms [17•] and amplify the innate immune response against $S$. aureus [18]. In addition, staphylococcal PSMs enhance the antimicrobial activity of host AMPs such as LL-37, CRAMP and hBDs, show chemotactic activity for host neutrophils and have proinflammatory properties. Thus, commensal derived small molecules may play roles in both immune defense and pathogenesis, and while being beneficial on intact skin, they may become pathogenic to the host when the interaction between commensals and host innate immunity is imbalanced.

S. epidermidis may also inhibit uncontrolled skin inflammation through a TLR2-TLR3-mediated cross talk mechanism [19]. After skin injury, host RNA from damaged cells activates TLR3 in keratinocytes, resulting in inflammation. Staphylococcal lipoteichoic acid in turn inhibits the inflammatory cytokine release from keratinocytes triggered by injury through a TLR2-dependent mechanism.

Skin microbiota promote the expression of components of the complement system, which is composed of a large number of proteins which react with one another to opsonize pathogens and induce inflammatory responses. In germ-free mice, the expression of the complement component $\mathrm{C} 5 \mathrm{aR}$ is impaired, resulting in decreased expression of antimicrobial peptides associated with dysbiosis of skin-resident microbes [20].
By influencing the local cytokine milieu in the skin and controlling in particular the level of expression of interleukin 1 (IL-1), skin commensals also modulate the function of T cells, resulting in increased potential to produce IL-17A and IFN $\gamma$ by dermal T cells and thereby enhancing skin immunity and defense against invading microbes. The healthy human skin is a large reservoir of memory T cells and like in the gut [21], a large fraction of these might be specific for commensals and therefore important for the reinforcement of skin immunity through IL-17 driven action on keratinocytes' antimicrobial function. In the gastrointestinal tract, microbe and products of microbial metabolisms may also promote immune homeostasis by promoting the induction of regulatory networks. A few examples suggest this may be true in the skin as well, with a recent study showing the epicutaneous exposure to Vitreoscilla filiformis can promote the induction of tissue resident $\mathrm{T}$ regulatory cells and inhibit $\mathrm{T}$ cell proliferation during cutaneous inflammation in mice. The balance between evading and stimulating immune defense and promoting immune homeostasis has important consequences for skin homeostasis.

\section{Association of the Microbiota With Skin Inflammatory Diseases}

Common skin disorders, such as atopic eczema, psoriasis, seborrheic dermatitis and acne, are associated with specific changes in the skin microbiome [22・•]. However, it has remained unclear whether this specificity is based primarily on the cutaneous microbiome or is only an epiphenomenon due to secondary factors, e.g. changes in the skin barrier or immunologic function [23]. The characteristic changes in microbial colonization have increased the interest in developing new microbiome-aimed diagnostic methods and treatments.

The role of the skin microbiome has been most extensively investigated in atopic eczema [24•]. Some recent studies have pointed towards a possible underlying microbial etiology, and clinical improvement has been seen with antimicrobial treatments [25•]. Changes in the cutaneous microbiome and the host's cutaneous immune response have been shown to exacerbate atopic dermatitis and lead to secondary skin infections. During disease flares, patients with atopic dermatitis have showed a decrease of cutaneous microbial diversity, which regenerates after sufficient therapy (Fig. 2). Interestingly, the microbiome shifts have been primarily confined to sites of disease predilection, e.g. folds or facial area.

Recognized effective treatments in atopic dermatitis (topical corticosteroids, topical calcineurin inhibitors and basic emollients) have been shown to diversify the skin microbiome. Treatment-associated increases in microbial diversity may be based on therapies that specifically decrease Staphylococcus aureus colonization and subsequently 
Disease under treatment, diverse microbiome (neck)

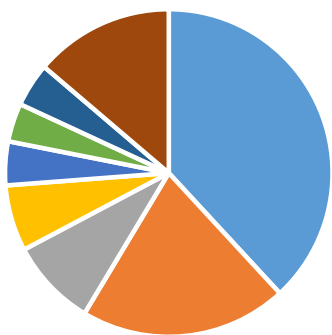

- Proprionibacteria spp. = Coruynebacterium spp - Lactococcus spp. " Staphylococcus spp. - Prevottella spp. = Enhydrobacter spp. . - Streptococcus spp. - Other taxa

Disease flare, reduced microbial diversity, Staphylococcus spp. predominance (neck)

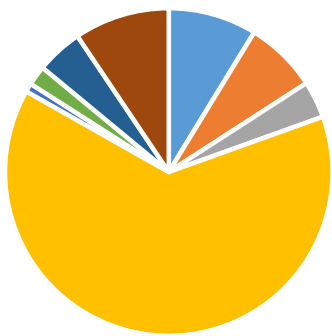

- Proprionibacteria spp. - Coruynebacterium spp = Lactococcus spp. - Staphylococcus spp. - Prevottella spp. - Enhydrobacter spp. - Streptococcus spp. = Other taxa

Fig. 2 Mean relative abundances of the seven most common genera of the cutaneous microbiome in atopic dermatitis during stable disease phase and disease flares

permitting the growth of apathogenic bacteria. A possible other explanation could be in the selection of microbes that control Staphylococcus aureus prevalence or a general reduction of skin microbes, which is followed by a regeneration of broader diversity [26].

Particular attention has been paid to the role of Staphylococcus aureus in inflammatory skin disorders. Some evidence is emerging, e.g. that during flares of atopic dermatitis the skin microbiome is altered to more susceptible towards Staphylococcus aureus colonization. In flares, an increase of otherwise commensal Staphylococcus epidermidis and an appearance of Staphylococcus aureus colonization has been a frequent observation. Additionally, variations in the skin microbiome have been shown to modify the interaction of genes and environment on the cutaneous surface and thus may have an impact on the skin's immune defense. Recently, an example has been described how host and apathogenic cutaneous microbial flora collaborate against pathogenic microbes [27•]. Here, the usually apathogenic Staphylococcus epidermidis showed to inhibit pathogenic Staphylococcus aureus colonization and biofilm formation on the skin.

Inflammatory skin disorders often present in typical localizations and atopic dermatitis can be considered as a prime example. This specificity to sites proposes, that not only do specific microbial niches of the skin cause the growth of certain bacteria, but that the skin microbiome is important in the initiation and progression of the disease [28].

Microorganisms and the skin microbiome play a secondary role in many inflammatory skin disorders, but there is only limited understanding about their contribution to the clinical picture and role in pathogenesis. Many important questions about the host-microbiome relationship and its relevance still remain unclear.

\section{Future Therapeutic Opportunities}

The cutaneous microbiome in inflammatory skin disorders has been investigated broadly in recent years and some traditional perspectives are beginning to change. A diverse microbiome exists on human skin and has a potential to influence pathogenic microbes and modulate the course of skin disorders, e.g. atopic dermatitis. In addition to the known dysfunctions in barrier function of the skin and immunologic disturbances, evidence is rising that frequent skin disorders, e.g. atopic dermatitis, might be connected to a dysbiosis of the microbial community and changes in the skin microbiome.

The changes in the cutaneous microbiome have been quite characteristic for a specific disease state (stable or exacerbated) and as prognostic factors (disease development) and therefore may constitute a potential diagnostic tool in the future.

Molecular techniques used to investigate the skin microbiome have proved to be sensitive and less-biased and could therefore be used supplementary to clinical and microscopic diagnostics. As a future perspective, examining the skin microbiome could be seen as a potential new diagnostic and therapeutic target in inflammatory skin disorders, e.g. atopic dermatitis [29].

\section{Conclusion and Perspectives}

Molecular genetics have dramatically changed our vision of the microorganisms colonizing the human skin. Important new questions have arisen in skin disorders such as atopic dermatitis: What relationship does the host microorganisms interaction have to pathogenesis? What is the dysbiosis concept? What is the role of anti-microbial treatments?

While some of the main dominant skin microbes, e.g. Staphylococcus spp., have been investigated widely, we have only limited understanding of rare and transient microbes. In addition, it remains widely unclear what factors are causing the changes in skin microbiome during flares of inflammatory skin diseases such as atopic dermatitis.

A new understanding of the skin microbiome is emerging, and compared to our previous concepts, the cutaneous microbiome has shown to be more complex and interesting. Future studies may answer various aspects of the pathogenesis 
of inflammatory skin diseases and accordingly, the examination of the patient's skin microbiome may have a role in diagnostics and treatments of skin disorders.

\section{Compliance with Ethical Standards}

Conflict of Interest Drs. Fyhrquist, Salava, Auvinen, and Lauerma declare no conflicts of interest.

Human and Animal Rights and Informed Consent This article does not contain any studies with human or animal subjects performed by any of the authors.

\section{References}

Papers of particular interest, published recently, have been highlighted as:

- Of importance

•- Of major importance

1. Brestoff JR, Artis D. Commensal bacteria at the interface of host metabolism and the immune system. Nat Immunol. 2013;14:67684. Comprehensive review about the cutaneous microbiome and microbe-microbe dynamics.

2.• Belkaid Y, Segre JA. 2014. Science. 2014;346:954-9. Good and recent review about host-microbe interplay.

3.• Grice EA, Segre JA. The skin microbiome. Nat Rev Microbiol. 2011;9:244-53. Up-to date article about new methods exploring the skin microbiome and future prespectives.

4. Oh J, Byrd AL, Deming C, Conlan S, Program NCS, Kong HH, et al. Biogeography and individuality shape function in the human skin metagenome. Nature. 2014;514:59-64.

5. Sharon G, Garg N, Debelius J, Knight R, Dorrestein PC, Mazmanian SK. Specialized metabolites from the microbiome in health and disease. Cell Metab. 2014;20:719-30.

6. Pasparakis M, Haase I, Nestle FO. Mechanisms regulating skin immunity and inflammation. Nat Rev Immunol. 2014;14:289-301.

7. Nakatsuji T, Chiang HI, Jiang SB, Nagarajan H, Zengler K, Gallo RL. The microbiome extends to subepidermal compartments of normal skin. Nat Commun. 2013;4:1431.

8. Wilson M. Microbial inhabitants of humans: their ecology and role in health and disease. 2005. Cambridge University Press.

9. Picardo M, Ottaviani M, Camera E, Mastrofrancesco A. Sebaceous gland lipids. Dermatoendocrinol. 2009;1:68-71.

10. Scott JC, Sahl HG, Carne A, Tagg JR. Lantibiotic-mediated antilactobacillus activity of a vaginal Staphylococcus aureus isolate. FEMS Microbiol Lett. 1992;72:97-102.

11. Otto M, Sussmuth R, Vuong C, Jung G, Gotz F. Inhibition of virulence factor expression in Staphylococcus aureus by the Staphylococcus epidermidis agr pheromone and derivatives. FEBS Lett. 1999;450:257-62.

12. Mazmanian SK, Round JL, Kasper D. A microbial symbiosis factor prevents intestinal inflammatory disease. Nature. 2008;453:620-5.

13. Maslowski KM, Vieira AT, Ng A, Kranich J, Sierro F, Yu D, et al. Regulation of inflammatory responses by gut microbiota and chemoattractant receptor GPR43. Nature. 2009;461:1282-6.

14. Ushijima T, Takahashi M, Ozaki Y. Acetic, propionic, and oleic acid as the possible factors influencing the predominant residence of some species of Propionibacterium and coagulase-negative Staphylococcus on normal human skin. Can J Microbiol. 1984;30:647-52.

15. Shu M, Wang Y, Yu J, Kuo S, Coda A, Jiang Y, et al. Fermentation of Propionibacterium acnes, a commensal bacterium in the human skin microbiome, as skin probiotics against methicillin-resistant Staphylococcus aureus. PLoS One. 2013;8:e55380.

16. Nakatsuji T, Kao MC, Zhang L, Zouboulis CC, Gallo RL, Huang CM. Sebum free fatty acids enhance the innate immune defense of human sebocytes by upregulating beta-defensin-2 expression. J Invest Dermatol. 2010;130:985-94.

17. Lai Y, Cogen AL, Radek KA, Park HJ, Macleod DT, Leichtle A, et al. Activation of TLR2 by a small molecule produced by Staphylococcus epidermidis increases antimicrobial defense against bacterial skin infections. J Invest Dermatol. 2010;130:2211-21. Good study about the cutaneous microbe-microbe interplay and dynamics.

18. Wanke I, Steffen H, Christ C, Krismer B, Gotz F, Peschel A, et al. Skin commensals amplify the innate immune response to pathogens by activation of distinct signaling pathways. J Invest Dermatol. 2011;131:382-90.

19. Lai Y, Di Nardo A, Nakatsuji T, Leichtle A, Yang Y, Cogen AL, et al. Commensal bacteria regulate Toll-like receptor 3-dependent inflammation after skin injury. Nat Med. 2009;15:1377-82.

20. Chehoud C, Rafail S, Tyldsley AS, Seykora JT, Lambris JD, Grice EA. Complement modulates the cutaneous microbiome and inflammatory milieu. Proc Natl Acad Sci. 2013;110:15061-6.

21. Yang Y, Torchinsky MB, Gobert M, Xiong H, Xu M, Linehan JL, et al. Focused specificity of intestinal TH17 cells towards commensal bacterial antigens. Nature. 2014;510:152-6.

22.• Zeeuwen PL, Kleerebezem M, Timmerman HM, Schalkwijk J. Microbiome and skin diseases. Curr Opin Allergy Clin Immunol. 2013;13(5):514-20. Recent and well-done review about cutaneous microbiome and inflammatory skin diseases.

23. Seite $\mathrm{S}$, Bieber T. Barrier function and microbiotic dysbiosis in atopic dermatitis. Clin Cosmet Investig Dermatol. 2015;15(8): 479-83.

24. Salava A, Lauerma A. Role of the skin microbiome in atopic dermatitis. Clin Transl Allergy. 2014;17(4):33. Comprehensive and recent review about the cutaneous microbiome and atopic dermatitis.

25. Kong HH, Oh J, Deming C, Conlan S, Grice EA, Beatson MA, et al. Temporal shifts in the skin microbiome associated with disease flares and treatment in children with atopic dermatitis. Genome Res. 2012;22(5):850-9. Good clinical study about the microbiome dynamics in atopic dermatitis and decrease of microbial diversity during flares of the skin disease.

26. Williams MR, Gallo RL. The role of the skin microbiome in atopic dermatitis. Curr Allergy Asthma Rep. 2015;15(11):65.

27. Iwase T, Uehara Y, Shinji H, Tajima A, Seo H, Takada K, et al. Staphylococcus epidermidis Esp inhibits Staphylococcus aureus biofilm formation and nasal colonization. Nature. 2010;465:3469. Study showing good examples about the microbe-microbe dynamics and antagonism of commensal bacteria with pathogens.

28. Baviera G, Leoni MC, Capra L, Cipriani F, Longo G, Maiello N, et al. Microbiota in healthy skin and in atopic eczema. Biomed Res Int. 2014;2014:436921

29. Powers CE, McShane DB, Gilligan PH, Burkhart CN, Morrell DS. Microbiome and pediatric atopic dermatitis. J Dermatol. 2015;42(12):1137-42.

30. Salava A, Aho V, Pereira P, Koskinen K, Paulin L, Auvinen P, et al. Skin microbiome in melanomas and melanocytic nevi. Eur $\mathrm{J}$ Dermatol. 2016;26(1):49-55. 